\title{
Strain Measurement through the Thickness of Crystal using DBI
}

\author{
Mana Norouzpour, Rodney Herring
}

CAMTEC, Mechanical Engineering/University of Victoria, Victoria, Canada.

Accurate measurement of strain, which plays an important role in physical properties of materials, has been the subject of intense works for decades. Moreover, knowing the strain in 3D at the atomic scale is highly desired for crystal growers and microelectronic device manufacturers since it creates undesirable defects that destroy the beneficial properties of the crystal, however positively, strain can enhance a device's performance by increasing the electron and hole mobility. Here we report a technique of DBI (Diffracted Beam Interferometry) that enables recovering the phase information, which is lost during recording by detectors. The retrieved phase provides information to measure the strain distribution through the thickness of crystals that can be compared to simulated strain profiles providing a method to determine the three dimensional strain distribution. As well, it is known that Higher Order Laue Zone (HOLZ) intensities have sufficient coherence to form high contrast fringes when self-interfered [1, 2]. HOLZ lines are well known for measuring strain with a remarkable sensitivity of $10^{-4}$. In combination with DBI, they complement the developed method of CBED (Convergent Beam Electron Diffraction) used to map the direction and relative magnitude of the displacement field by fitting the Hough transforms of experimental and kinematically simulated HOLZ lines [3].

DBI involves self- interference of split HOLZ lines by applying a controlled range of positive voltages from $0 \mathrm{~V}$ to $\sim 30 \mathrm{~V}$ on the electron optically focused biprism inserted in between the specimen and diffraction plane (Fig. 1a). The split HOLZ line within the 000 disc located close to the [320] zone axis, is formed from a Si substrate near the $S i_{0.7} G e_{0.3} / S i$ interface and the interference fringes running parallel to the line direction of the split HOLZ line (Fig 1b-1c). The experimental details include a JEOL JEM-2010F operated at $200 \mathrm{kV}$, a probe of $5 \mathrm{~nm}$, specimen thickness of $\sim 200 \mathrm{~nm}$, energy filtered electrons using a GIF Tridiem and the zero loss electrons having a $5 \mathrm{eV}$ window. The measured phase profile across the split HOLZ line was obtained using Fourier reconstruction steps by means of Holoworks, a DigitalMicrograph subroutine, indicating a broad peak between 2 lower intensity peaks on either side (Fig. 2a). Simulations of possible phase profiles having displacement fields, $R$, about the Si/SiGe interface, include a $V$-shape type, a parabola type and a bell-shape type [3], were performed. Only the phase profile of the bell-shape type and the experimental phase profile (Fig. 2b) fit well. Subsequently, with respect to the lattice parameter of the substrate $S i$ or epitaxial layer $S i_{0.7} G e_{0.3}$, the strain profile through the thickness of the crystal has been determined for the first time (Fig. 2c). Considering the relative direction and magnitude of the displacement field while moving away from the $\mathrm{Si} / \mathrm{SiGe}$ interface into the substrate [3], allows determination of the three-dimensional (3D) strain within the crystal (Fig. 2d). This technique can be applied to the large number of split HOLZ lines present in the diffraction pattern that represent distinct sets of bent lattice planes enabling all of the six strain tensors to be measured. Thus, the self-interference of split HOLZ lines takes the current strain measurement capability from $2 \mathrm{D}$ to full $3 \mathrm{D}$ crystal strain determination.

1. R. A. Herring et al, M\&M, (2011), p.1232-1233.

2. R. A. Herring, J. Electron Microscopy, 58(3) (2009), p. 213-221.

3. Koh Saitoh et al, J. Electron Microscopy, 59(5) (2010), p. 367-378. 

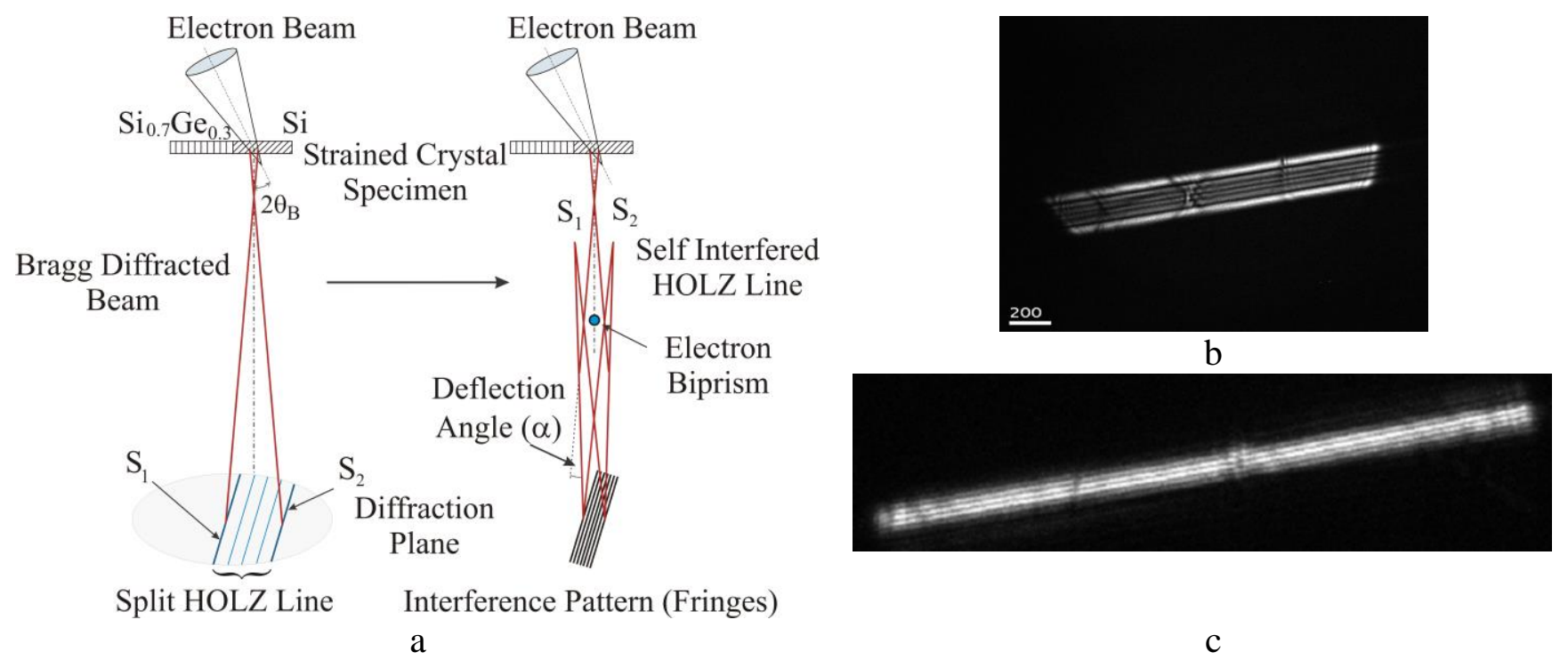

Figure 1. a) Schematic of DBI technique, b) Experimental split HOLZ line in the dark field mode, c) the formed interference fringes by DBI parallel to HOLZ line.

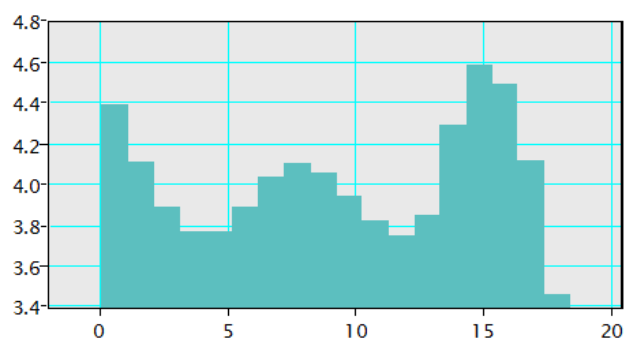

a

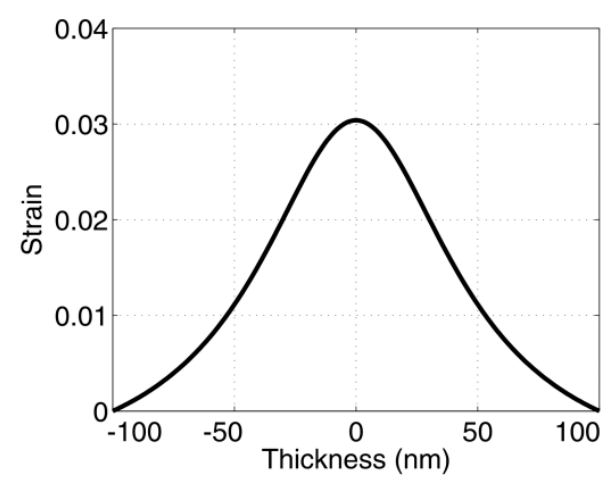

c

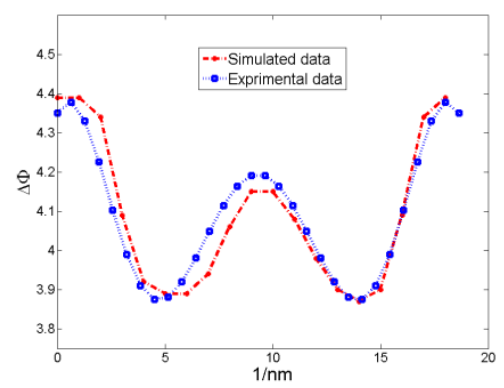

b

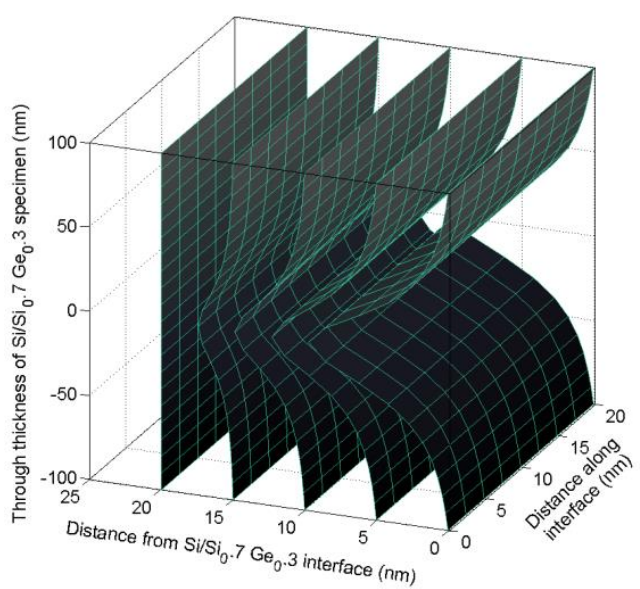

d

Figure 2. a) Recovered phase profile across the split HOlZ line, b) bell-shaped simulated phase profile fits perfectly the experimental phase profile, c) strain plot through the thickness of the crystal using (b), d) 3D strain distribution within the crystal away from the $\mathrm{Si} / \mathrm{Si}_{0.7} G e_{0.3}$ interface. 